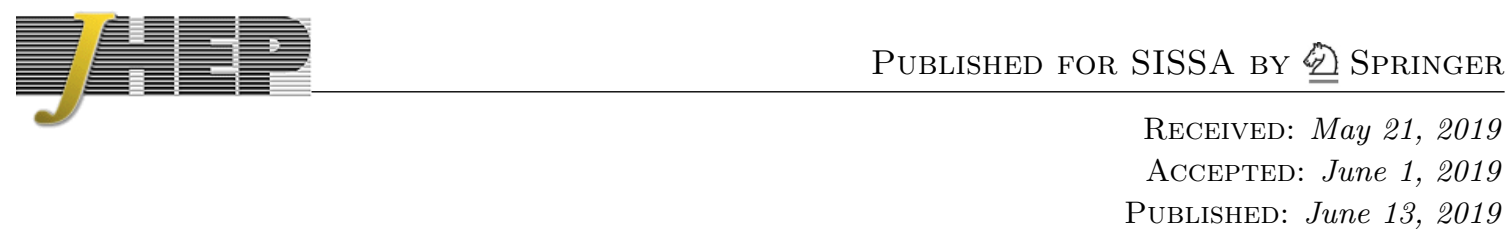

\title{
Trapping horizon and negative energy
}

\author{
Pei-Ming $\mathrm{Ho}^{a}$ and Yoshinori Matsuo ${ }^{b}$ \\ ${ }^{a}$ Department of Physics and Center for Theoretical Physics, \\ National Taiwan University, Taipei 106, Taiwan, R.O.C. \\ ${ }^{b}$ Department of Physics, Osaka University, \\ Toyonaka, Osaka 560-0043, Japan \\ E-mail: pmho@phys.ntu.edu.tw, matsuo@het.phys.sci.osaka-u.ac.jp
}

ABSTRaCt: Assuming spherical symmetry and the semi-classical Einstein equation, we prove that, for the observers on top of the trapping horizon, the vacuum energy-momentum tensor is always that of an ingoing negative energy flux at the speed of light with a universal energy density $\mathcal{E} \simeq-1 /\left(2 \kappa a^{2}\right)$, (where $a$ is the areal radius of the trapping horizon), which is responsible for the decrease in the black hole's mass over time. This result is independent of the composition of the collapsing matter and the details of the vacuum energy-momentum tensor. The physics behind the universality of this quantity $\mathcal{E}$ and its surprisingly large magnitude will be discussed.

Keywords: Black Holes, Effective Field Theories

ARXIV EPRINT: 1905.00898 


\section{Contents}

1 Introduction 1

2 Einstein equations around trapping horizon $\quad 2$

2.1 Trapping horizon 3

2.2 Expansion around trapping horizon 4

3 Magnitude of quantum corrections $\quad 6$

4 Energy-momentum tensor on trapping horizon 7

4.1 A universal result 8

$\begin{array}{lll}4.2 & \text { Connection to surface gravity } & 10\end{array}$

5 Comments 11

$\begin{array}{ll}\text { A An alternative scenario } & 12\end{array}$

\section{Introduction}

The spacetime geometry for the dynamical process of a gravitational collapse is hard to solve analytically from the semi-classical Einstein equation if the back reaction of the vacuum energy-momentum tensor is included. The spacetime region far away from the black hole can be well described by the outgoing Vaidya metric (including the back reaction of the Hawking radiation), and the region well inside the collapsing matter by the ingoing Vaidya metric (ignoring the vacuum energy-momentum tensor). But the geometry of a neighborhood of the horizon has only been studied numerically or with various approximations.

For instance, for the vacuum energy-momentum tensor given by the DFU model [1], the numerical analysis was carried out in ref. [2], and the analytic solution was computed in ref. [3] with the assumption of weak time-dependence.

In this work, we zoom into the neighborhood of the trapping horizon and study the implication of its geometric properties on the energy-momentum tensor through the semiclassical Einstein equation, assuming spherical symmetry for simplicity. There is no assumption about the details of the vacuum energy-momentum tensor except the implicit assumption of an $\hbar$-expansion with the absence of the zero-th order (classical) term (see eqs. (3.2) and (3.4) below). Surprisingly, a universal expression (eq. (4.9)), which is uniquely determined by the black-hole mass, applies to the vacuum energy-momentum tensor at the trapping horizon. It corresponds to a light-like ingoing negative energy flux with the energy density (eq. (4.10))

$$
T_{\xi \xi} \simeq-\frac{1}{2 \kappa a^{2}(u)}
$$

where $\xi$ is the normalized tangent vector on the trapping horizon. 
Apart from the fact that a universal expression of the energy-momentum tensor is derived, another surprise is that the expression above (1.1) diverges in the limit $\hbar \rightarrow 0$. Correspondingly, the Einstein tensor

$$
G_{\xi \xi} \sim \mathcal{O}\left(\frac{1}{a^{2}}\right)
$$

does not vanish in the limit $\hbar \rightarrow 0$. (Recall that the Einstein tensor vanishes identically for the Schwarzschild metric.) Rather, $G_{\xi \xi}$ has a magnitude of the same order (but opposite in sign) as the region occupied by ordinary matter. We will explain below how this seemingly paradoxical result is in fact compatible with the $\hbar$-expansion of the semi-classical Einstein equation.

The same result about the vacuum energy-momentum tensor was first obtained in a previous work [4], which was restricted to the conventional model of black holes and only the ingoing negative energy flux was included in the vacuum energy-momentum tensor as an approximation. This work is its generalization to a much greater class of models.

In section 2, we solve the semi-classical Einstein equation ${ }^{1}$ via a coordinate expansion around the trapping horizon. The magnitudes of the quantum corrections to relevant geometric quantities are estimated in section 3, which lead to the universal results about the vacuum energy-momentum tensor observed on the trapping horizon in section 4 . In section 5, we comment on the implications of this result to the problem of the holographic principle.

\section{Einstein equations around trapping horizon}

Assuming spherical symmetry, the most general metric in $4 \mathrm{D}$ is of the form

$$
d s^{2}=-C(u, v) d u d v+r^{2}(u, v) d \Omega^{2},
$$

where $d \Omega^{2}=d \theta^{2}+\sin ^{2} \theta d \phi^{2}$ is the metric on a 2-sphere. This metric (2.1) does not uniquely fix the choice of coordinates. A transformation such as

$$
u \rightarrow u^{\prime}(u), \quad v \rightarrow v^{\prime}(v)
$$

does not change the form of the metric $(2.1)$, with $C(u, v)$ transforming as

$$
C(u, v) \rightarrow C^{\prime}\left(u^{\prime}, v^{\prime}\right)=C(u, v)\left(\frac{d u^{\prime}}{d u}\right)^{-1}\left(\frac{d v^{\prime}}{d v}\right)^{-1}
$$

This is the residual gauge symmetry of diffeomorphism for the gauge condition in which the metric is of the form (2.1).

\footnotetext{
${ }^{1}$ By semi-classical Einstein equation, we mean that the energy-momentum tensor of the vacuum does not have to vanish, and that the weak energy condition can be violated.
} 


\section{$2.1 \quad$ Trapping horizon}

The spacetime with spherical symmetry is foliated by symmetric 2-spheres labelled by the coordinates $(u, v)$. A symmetric 2-sphere $S$ is a trapped surface if both outgoing and ingoing null geodesics normal to $S$ have negative expansions, that is,

$$
\partial_{v} r(u, v)<0 \quad \text { and } \quad \partial_{u} r(u, v)<0 .
$$

A 4D continuum of trapped surfaces form a trapped region, and its boundary is a trapping horizon. A space-like slice of the trapping horizon, which is a marginally trapped surface, is called an apparent horizon.

For the space outside the trapped region, we assume that

$$
\partial_{v} r(u, v)>0 \quad \text { and } \quad \partial_{u} r(u, v)<0
$$

(the same as in Minkowski space). Here, we assume that the geometry is asymptotically flat, or has the same property (2.5) as the Minkowski sapce in the asymptotic region. Since by definition the areal radius cannot be negative, in principle eq. (2.5) is always satisfied at $r=0$ as long as the trajectory of the origin is time-like. This means that at a given instant of time, there are two apparent horizons as two concentric 2-spheres [5-7].

As eq. (2.4) is satisfied inside the trapping horizon while eq. (2.5) holds outside, we must have

$$
\partial_{v} r=0
$$

on top of the trapping horizon.

As a finite closed space, the trapping horizon has maximal and minimal values of their $u, v$ coordinates. Let us denote by $A$ and $C$ the points with the minimal and maximal values of the $u$-coordinate, respectively. (See figure 1 [5-7].) Similarly, we denote by $B$ and $D$ the points on the trapping horizon with the maximal and minimal values of the $v$-coordinate.

We will specify an open segment of the trapping horizon as $A B, A B C$, etc., where $A B$ refers to the segment on the trapping horizon between the points $A$ and $B$, and $A B C$ refers to the segment from $A$ to $C$ through $B$. The segment $D A B$ is called the outer trapping horizon, and $B C D$ the inner trapping horizon in ref. [7].

Due to eqs. (2.4) and (2.5), we have

$$
\begin{array}{lll}
A B: & \partial_{v}^{2} r>0, & \partial_{u} \partial_{v} r<0, \\
B C: & \partial_{v}^{2} r>0, & \partial_{u} \partial_{v} r>0, \\
C D: & \partial_{v}^{2} r<0, & \partial_{u} \partial_{v} r>0, \\
D A: & \partial_{v}^{2} r<0, & \partial_{u} \partial_{v} r<0 .
\end{array}
$$

Exceptions are the degenerate cases in which the 2nd derivatives of $r$ vanishes, and the inequalities hold for higher derivatives of $r .^{2}$

\footnotetext{
${ }^{2}$ For instance, the condition $\partial_{v}^{2} r>0$ on the segment $A B$ can be replaced by$$
\partial_{v}^{2} r=\partial_{v}^{3} r=0, \quad \text { and } \quad \partial_{v}^{4} r>0 .
$$ 


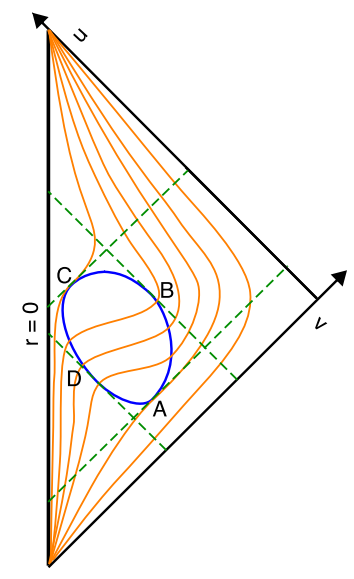

Figure 1. The schematic Penrose diagram with a trapping horizon. The trapping horizon is shown as the solid curve (in blue). The extremal points on the trapping horizon with the extrema of the coordinates $u$ and $v$ are marked as $A, B, C$ and $D$. The dashed lines (in green) are constant- $u$ and constant- $v$ lines tangent to the trapping horizon. The constant- $r$ curves (in orange) are tangent to constant- $u$ lines on the trapping horizon.

\subsection{Expansion around trapping horizon}

The trajectories $A B C$ and $A D C$ of the trapping horizon can be specified by their $v$ coordinates $v_{A B C}(u)$ and $v_{A D C}(u)$, respectively. We will use $v_{0}(u)$ to represent both $v_{A B C}(u)$ and $v_{A D C}(u)$. On a small neighborhood of the segment $A B C$ or $A D C$, we can expand the metric functions $C(u, v)$ and $r(u, v)$ in powers of $\left(v-v_{0}(u)\right)$ as

$$
\begin{aligned}
& C(u, v)=C_{0}(u)+C_{1}(u)\left(v-v_{0}(u)\right)+\frac{1}{2} C_{2}(u)\left(v-v_{0}(u)\right)^{2}+\frac{1}{6} C_{3}(u)\left(v-v_{0}(u)\right)^{3}+\cdots \\
& r(u, v)=a(u)+\frac{1}{2} r_{2}(u)\left(v-v_{0}(u)\right)^{2}+\frac{1}{6} r_{3}(u)\left(v-v_{0}(u)\right)^{3}+\frac{1}{24} r_{4}(u)\left(v-v_{0}(u)\right)^{4}+\cdots
\end{aligned}
$$

where the linear term in $r(u, v)$ vanishes because of the trapping horizon condition (2.6). According to eq. (2.13), $a(u)=r\left(u, v_{0}(u)\right)$ is the areal radius of the apparent horizon for a given $u$.

For the metric (2.1), the semi-classical Einstein equations are

$$
\begin{aligned}
G_{u u} & \equiv \frac{2 \partial_{u} C \partial_{u} r}{C r}-\frac{2 \partial_{u}^{2} r}{r}=\kappa T_{u u}, \\
G_{v v} & \equiv \frac{2 \partial_{v} C \partial_{v} r}{C r}-\frac{2 \partial_{v}^{2} r}{r}=\kappa T_{v v}, \\
G_{u v} & \equiv \frac{C}{2 r^{2}}+\frac{2 \partial_{u} r \partial_{v} r}{r^{2}}+\frac{2 \partial_{u} \partial_{v} r}{r}=\kappa T_{u v}, \\
G_{\theta \theta} & \equiv \frac{2 r^{2}}{C^{3}}\left(\partial_{u} C \partial_{v} C-C \partial_{u} \partial_{v} C\right)-\frac{4 r}{C} \partial_{u} \partial_{v} r=\kappa T_{\theta \theta},
\end{aligned}
$$


where the coupling constant $\kappa$ is related to the Newton constant $G_{N}$ via $\kappa=8 \pi G_{N}$. Due to spherical symmetry, we have $G_{\phi \phi}=G_{\theta \theta} \sin ^{2} \theta$ and $T_{\phi \phi}=T_{\theta \theta} \sin ^{2} \theta$. Here the energymomentum tensor is the full tensor including both classical and quantum contributions.

Solving the semi-classical Einstein equations (2.15) and (2.16) at the lowest order, we find

$$
\begin{aligned}
& r_{2}(u)=-\frac{1}{2} \kappa a(u) T_{v v}^{(0)}(u), \\
& \dot{v}_{0}(u)=\frac{C_{0}(u)-2 \kappa a^{2}(u) T_{u v}^{(0)}(u)}{-2 \kappa a^{2}(u) T_{v v}^{(0)}(u)}
\end{aligned}
$$

where

$$
T_{\mu \nu}^{(0)}(u) \equiv T_{\mu \nu}\left(u, v_{0}(u)\right)
$$

On the branch $A B C$, eqs. (2.7) and (2.8) imply that $r_{2}>0$, so that the ingoing energy flux must be negative

$$
T_{v v}^{(0)}(u)<0
$$

according to eq. (2.18). On the other hand, on the branch $A D C$, eqs. (2.9) and (2.10) imply that

$$
T_{v v}^{(0)}(u)>0
$$

It is therefore clear that the branch $A B C$ of the trapping horizon stays in vacuum and the other branch $A D C$ typically resides in matter. At the point $A$ where the two branches join (see figure 1), we have $T_{v v}^{(0)}=0$.

Furthermore, on the segment $D A B$, eqs. (2.7) and (2.10) imply that $r_{2} \dot{v}_{0}>0$, which means that

$$
C_{0}(u)>2 \kappa a^{2}(u) T_{u v}^{(0)}(u) .
$$

The semi-classical Einstein equation $G_{u v}=\kappa T_{u v}$, and the spherical symmetry implies that $G_{u v}=\frac{C}{2 r^{2}} R_{\theta \theta}$, so this condition is also equivalent to the geometric condition

$$
R_{\theta \theta}<1
$$

which is typically assumed in vacuum as we will see below in eq. (3.2).

On the other hand, on the segment $B C D, r_{2} \dot{v}_{0}<0$, so that we need

$$
C_{0}(u)<2 \kappa a^{2}(u) T_{u v}^{(0)}(u),
$$

which is equivalent to

$$
R_{\theta \theta}>1 .
$$

To summarize, the four segments of the trapping horizon divided by the extrema of $u$ and $v$ coordinates are characterized as follows:

$$
\begin{array}{lll}
A B: & T_{v v}<0, & R_{\theta \theta}<1, \\
B C: & T_{v v}<0, & R_{\theta \theta}>1, \\
A D: & T_{v v}>0, & R_{\theta \theta}<1, \\
D C: & T_{v v}>0, & R_{\theta \theta}>1 .
\end{array}
$$




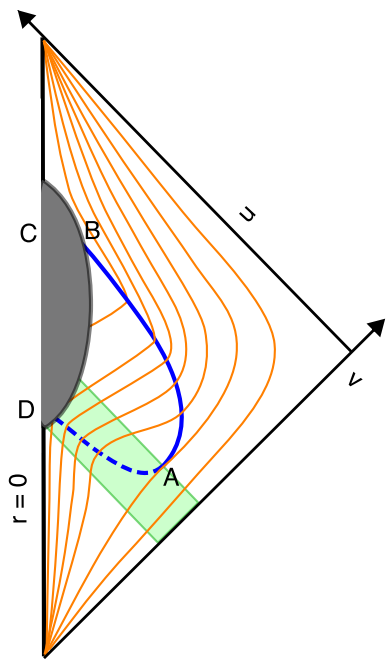

Figure 2. The Penrose diagram with a trapping horizon. The part of the trapping horizon in vacuum is shown as a solid curve (in blue), and the part in matter as a dashed curve (in blue), with the diagonal stripe (in green) representing the collapsing matter. The gray area around the origin represents the high-curvature region behind which the points $B, C$ and $D$ in figure 1 are hidden. The constant- $r$ curves (in orange) are tangent to constant- $u$ lines on the trapping horizon.

Recall that the Schwarzschild solution has $R_{\theta \theta}=0$, so $\left|R_{\theta \theta}\right| \ll 1$ is expected for an "uneventful" horizon in vacuum. While the segment $C D$ is expected to be completely inside the collapsing matter where the matter density can be very large to produce a large spacetime curvature, the exceptionally large $R_{\theta \theta}$ on the segment $B C$ should be limited to the late stage of the evaporation when the size of the black hole is small and the Hawking radiation becomes comparable to classical matter, or when the collapsed matter bounces back. Hence we modify the Penrose diagram with trapping horizon in figure 1 to figure 2 . There, the segment $B C D$ is hidden in a region (the gray area) where either the collapsing matter has a large energy density around the origin, or the vacuum energy-momentum tensor is large at the last stage of the evaporation.

For convenience, we will refer to the branch of the trapping horizon represented by the solid curve in figure 2 as "the outer trapping horizon in vacuum", and the branch represented by the dashed curve in figure 2 as "the outer trapping horizon in matter".

\section{Magnitude of quantum corrections}

Without specifying the details of the vacuum energy-momentum tensor, it is often assumed that, for a large black hole, the horizon does not look too different from the Minkowski vacuum. For example, for a black hole with the Schwarzschild radius $a$, one expects the scalar curvature $R \ll \mathcal{O}\left(1 / a^{2}\right)$. Throughout this paper, we shall assume that

$$
\kappa \ll a^{2} .
$$

In vacuum, the energy-momentum tensor involves a factor of $\hbar$, and the factor $\hbar$ appears in the semi-classical Einstein equation $G_{\mu \nu}=\kappa T_{\mu \nu}$ only through its product with 
the Newton constant $\kappa$. We can thus adopt the convention that $\hbar=1$ and count the order of $\hbar$ in the $\hbar$-expansion in terms of the order of $\kappa$ in the $\kappa$-expansion.

For the Schwarzschild metric, an invariant geometric quantity of dimension $n$ is expected to be of order $\mathcal{O}\left(1 / a^{n}\right)$ as the Schwarzschild radius $a$ is the only quantity with nontrivial dimension, and its quantum correction should be of order $\mathcal{O}\left(\kappa / a^{n+2}\right)$ or less. For geometric quantities that are exactly 0 for the Schwarzschild metric (e.g. the scalar curvature), we can estimate their magnitudes by their quantum corrections.

For example, on the trapping horizon in vacuum (the solid blue curve in figure 2),

$$
\begin{aligned}
\left|R_{\theta \theta}\right| & \lesssim \mathcal{O}\left(\frac{\kappa}{a^{2}}\right) \ll 1, \\
\left|g^{u v} R_{u v}\right| & \lesssim \mathcal{O}\left(\frac{\kappa}{a^{4}}\right) \ll \mathcal{O}\left(\frac{1}{a^{2}}\right), \\
\left|\operatorname{det} R^{(2)}\right| & \lesssim \mathcal{O}\left(\frac{\kappa}{a^{6}}\right) \ll \mathcal{O}\left(\frac{1}{a^{4}}\right),
\end{aligned}
$$

where $R^{(2)}$ is the $2 \mathrm{D}$-matrix of the Ricci tensor

$$
R^{(2)} \equiv\left(\begin{array}{cc}
R^{u}{ }_{u} & R^{u}{ }_{v} \\
R^{v}{ }_{u} & R^{v}{ }_{v}
\end{array}\right) .
$$

Since $G_{\mu \nu} \equiv R_{\mu \nu}-\frac{1}{2} g_{\mu \nu} R$, and the Ricci scalar is $R \equiv 2 g^{u v} R_{u v}+2 g^{\theta \theta} R_{\theta \theta}$ due to the spherical symmetry, the conditions above are equivalent to

$$
\begin{aligned}
\left|2 \kappa a^{2}(u) T_{u v}^{(0)}(u)\right| & \ll C\left(u, v_{0}(u)\right), \\
\left|\kappa T_{\theta \theta}^{(0)}(u)\right| & \ll 1, \\
\left|4 \kappa^{2} a^{4}(u) T_{u u}^{(0)}\left(u, v_{0}(u)\right) T_{v v}^{(0)}(u)\right| & \ll C^{2}\left(u, v_{0}(u)\right) .
\end{aligned}
$$

These inequalities are invariant under the gauge transformation (2.2).

Eqs. (3.2) and (3.4) (or equivalently, eqs. (3.6) and (3.8)) are the only inequalities assumed in this work. In the conventional model of black holes, $T_{u u}$ and $T_{u v}$ are of order $\mathcal{O}\left(\kappa^{2} / a^{8}\right)$ and $\mathcal{O}\left(\kappa / a^{4}\right)$, respectively, at the horizon, and $\left|T_{v v}\right|$ is of order $\mathcal{O}\left(1 / a^{4}\right)$, so these assumptions are satisfied. But we emphasize that our assumptions are weaker than the conventional model. For instance, we do not need to assume that $\left|T_{v v}\right|$ is much larger than $\left|T_{u u}\right|$.

\section{Energy-momentum tensor on trapping horizon}

In the following, we focus on the spacetime geometry in a small neighborhood of the outer trapping horizon, i.e. the segment $D A B$ in figure 2 .

Define a time-like vector on the trapping horizon by

$$
\xi(u)=\left(\xi^{u}(u), \xi^{v}(u)\right)=\lambda(u)\left(1,\left|\dot{v}_{0}(u)\right|\right)
$$


where

$$
\lambda(u)=\frac{1}{\sqrt{C_{0}\left|\dot{v}_{0}\right|}}
$$

is chosen such that $\xi(u)$ is of unit length. The normalized vector orthogonal to $\xi(u)$ in the $u-v$ plane is then

$$
\zeta(u)=\left(\zeta^{u}(u), \zeta^{v}(u)\right)=\lambda(u)\left(1,-\left|\dot{v}_{0}(u)\right|\right) .
$$

Together, $\xi(u)$ and $\zeta(u)$ compose an orthonormal basis of vectors on the trapping horizon. On the trapping horizon in vacuum, $\xi(u)$ is tangent to the trapping horizon. On the trapping horizon in matter, $\zeta(u)$ is tangent to the trapping horizon.

The energy density, energy flow and pressure for an observer on the trapping horizon are

$$
\begin{aligned}
T_{\xi \xi} & \equiv T_{\mu \nu}^{(0)} \xi^{\mu} \xi^{\nu}=\lambda^{2}\left[T_{u u}^{(0)}+2\left|\dot{v}_{0}\right| T_{u v}^{(0)}+\dot{v}_{0}^{2} T_{v v}^{(0)}\right] \\
& =\epsilon \frac{\left(C_{0}-2 \kappa a^{2} T_{u v}^{(0)}\right)\left(\left(C_{0}-2(1-2 \epsilon) \kappa a^{2} T_{u v}^{(0)}\right)+4 \kappa^{2} a^{4} T_{u u}^{(0)} T_{v v}^{(0)}\right.}{2 \kappa a^{2} C_{0}\left(C_{0}-2 \kappa a^{2} T_{u v}^{(0)}\right)}, \\
T_{\xi \zeta} & \equiv T_{\mu \nu}^{(0)} \xi^{\mu} \zeta^{\nu}=\lambda^{2}\left[T_{u u}^{(0)}-\dot{v}_{0}^{2} T_{v v}^{(0)}\right] \\
& =-\epsilon \frac{\left(C_{0}-2 \kappa a^{2} T_{u v}^{(0)}\right)^{2}-4 \kappa^{2} a^{4} T_{u u}^{(0)} T_{v v}^{(0)}}{2 \kappa a^{2} C_{0}\left(C_{0}-2 \kappa a^{2} T_{u v}^{(0)}\right)}, \\
T_{\zeta \zeta} & \equiv T_{\mu \nu}^{(0)} \zeta^{\mu} \zeta^{\nu}=\lambda^{2}\left[T_{u u}^{(0)}-2\left|\dot{v}_{0}\right| T_{u v}^{(0)}+\dot{v}_{0}^{2} T_{v v}^{(0)}\right] \\
& =\epsilon \frac{\left(C_{0}-2 \kappa a^{2} T_{u v}^{(0)}\right)\left(C_{0}-2(1+2 \epsilon) \kappa a^{2} T_{u v}^{(0)}\right)+4 \kappa^{2} a^{4} T_{u u}^{(0)} T_{v v}^{(0)}}{2 \kappa a^{2} C_{0}\left(C_{0}-2 \kappa a^{2} T_{u v}^{(0)}\right)}
\end{aligned}
$$

where $\epsilon$ is the sign of $T_{v v}^{(0)}$,

$$
\epsilon \equiv \frac{T_{v v}^{(0)}}{\left|T_{v v}^{(0)}\right|} .
$$

\subsection{A universal result}

According to the estimate (3.6), the value of $\dot{v}_{0}(u)(2.19)$ and the energy-momentum tensor (4.4)-(4.6) can be simplified as

$$
\begin{aligned}
\dot{v}_{0}(u) & \simeq-\frac{C_{0}(u)}{2 \kappa a^{2}(u) T_{v v}^{(0)}(u)}, \\
T_{\xi \xi} & \simeq-T_{\xi \zeta} \simeq T_{\zeta \zeta} \simeq \frac{\epsilon}{2 \kappa a^{2}}
\end{aligned}
$$

in the neighborhood of the trapping horizon.

The main result of this work is the following. First, for $T_{v v}<0$ in vacuum, we have $\dot{v}_{0}>0$ according to eq. (4.8), and so the outer trapping horizon in vacuum is time-like. More importantly, the vacuum energy-momentum tensor (4.9) for observers staying on top of the outer trapping horizon in vacuum is thus a light-like negative ingoing energy with the energy density

$$
\mathcal{E}_{-}(u) \equiv T_{\xi \xi} \simeq-\frac{1}{2 \kappa a^{2}(u)}
$$


which amounts to the power of

$$
\mathcal{P}_{-} \simeq-\frac{2 \pi}{\kappa}
$$

through the outer trapping horizon in vacuum.

Notice that, due to the spherical symmetry, $\mathcal{E}_{-}$is a gauge-invariant quantity, and it is independent of the details of the quantum field theory responsible for the vacuum energymomentum tensor or the initial state. It only depends on the assumption of spherical symmetry, the semi-classical Einstein equation, and the existence of the trapping horizon.

From the viewpoint of observers on the outer trapping horizon in vacuum, the mass of the black hole decreases not because of the outgoing Hawking radiation, but because of the ingoing negative energy flow $\mathcal{P}_{-}$.

Notice that the magnitude of the energy density (4.10) is exceptionally large for vacuum. How is it possible to have such a large quantum effect? Let us examine this issue more carefully.

For the classical Schwarzschild metric, the vacuum energy-momentum tensor $T_{\mu \nu}$ vanishes identically, hence one would expect that its quantum correction is of order $\mathcal{O}(\hbar)$. There is however a technical subtlety. The energy density $\mathcal{E}_{-}$is defined for observers sitting on top of the horizon. For the classical black hole, the horizon is light-like, so these observers are moving outward at the speed of light. As a result, there is an infinite Lorentz factor, so that the energy density $\mathcal{E}_{-}$is strictly speaking ill-defined, as a product of 0 and infinity.

If we compute the vacuum expectation value of the energy-momentum tensor for a certain quantum field in the Schwarzschild background, typically $T_{v v}$ is non-zero, and the energy density $\mathcal{E}_{-}$diverges at the horizon due to the infinite Lorentz factor. ${ }^{3}$

Our calculation above shows that, when the back reaction of the vacuum energymomentum tensor is taken into consideration so that the Schwarzschild metric is modified, the energy density $\mathcal{E}_{-}$is "regularized" from infinity to a finite value given by eq. (4.10). It is therefore not unreasonable to see that it diverges in the limit $\kappa \rightarrow 0$.

Local gauge-invariants such as $T_{\mu}^{\mu}$ are still small, vanishing in the classical limit $\hbar \rightarrow 0$. The energy density $\mathcal{E}_{-}$is a non-local invariant. ${ }^{4}$ However, recall that Hawking radiation is also described by a nonlocal term in the effective action.

Similarly, since $T_{v v}>0$ inside the collapsing matter, we have $\dot{v}_{0}<0$ according to eq. (4.8), and so the outer trapping horizon in matter is space-like. The matter energymomentum tensor defined in the (gauge-invariant) reference frame of $(\xi, \zeta)$ is a light-like positive ingoing energy with the energy density

$$
\mathcal{E}_{+}(u) \simeq \frac{1}{2 \kappa a^{2}(u)}
$$

\footnotetext{
${ }^{3}$ It should be noted that the divergence does not appear on the future horizon but on the past horizon and at the intersection of two horizons.

${ }^{4} \mathcal{E}_{-}$is not a local gauge-invariant as it demands the information about the trapping horizon, which is uniquely defined only when there is spherical symmetry, and the spherical symmetry requires nonlocal information about the spacetime geometry.
} 
which leads to the power of

$$
\mathcal{P}_{+} \simeq \frac{2 \pi}{\kappa}
$$

through the outer trapping horizon in matter.

\subsection{Connection to surface gravity}

The reader may find it surprising that the vacuum energy density observed on the outer trapping horizon in vacuum is given by a universal formula (4.10) independent of how we define the quantum field theory responsible for the vacuum energy. The same result holds for any given energy-momentum tensor $T_{\mu \nu}$ as long as the trapping horizon exists. Such a robustness of the energy density on the horizon is possible for the following reason. If we imagine a modification to the energy-momentum tensor due to a change in the quantum field theory or a change in the initial state, the slope $d v / d u$ (4.8) would change accordingly, so that the trajectory of the trapping horizon is also changed. As $T_{\xi \xi}$ is defined with respect to the tangent vector $\xi$ of the trapping horizon, it is possible that the changes in $\xi$ and $T_{\mu \nu}$ cancel to keep the value of $T_{\xi \xi}$ invariant.

The comment above explains how it is possible for $T_{\xi \xi}$ to keep a constant value independent of all sorts of variations of the underlying theory or states, but it does not explain why it is given by the specific value (4.10). To address the latter issue, we comment on the relation between $T_{\xi \xi}$ and the surface gravity.

Using the Raychaudhuri equation, we have

$$
\kappa T_{\xi \xi} \simeq R_{\xi \xi}=-\left(D^{\mu} \xi^{\nu}\right)\left(D_{\nu} \xi_{\mu}\right)-\xi^{\mu} D_{\mu}\left(D_{\nu} \xi^{\nu}\right)+D_{\nu}\left(\xi^{\mu} D_{\mu} \xi^{\nu}\right)
$$

The second term on the right hand side is the time evolution of the expansion $D_{\nu} \xi^{\nu}$ along the trapping horizon, and the third term would vanish if $\xi$ is the tangent vector of a geodesic (although $\xi$ is not a geodesic here). Notice that, if $\xi$ is the Killing vector at the Killing horizon of a static black hole, both the second and third terms would vanish, and the first term is related to the surface gravity. Recall that the surface gravity $\kappa_{H}$ for a static black hole is expressed in terms of the Killing vector $\xi$ as

$$
\kappa_{H}^{2}=-\frac{1}{2}\left(D^{\mu} \xi^{\nu}\right)\left(D_{\mu} \xi_{\nu}\right)=\frac{1}{2}\left(D^{\mu} \xi^{\nu}\right)\left(D_{\nu} \xi_{\mu}\right),
$$

which is proportional to the first term on the right hand side of eq. (4.14). For the Schwarzschild solution, the apparent horizon is identical to the event horizon and the surface gravity is given by $\kappa_{H}=1 / 2 a$. Thus the right hand side of (4.14) gives the same universal result for the Schwarzschild solution as $\xi$ is replaced by the generator of the Killing horizon.

That is, the universal result comes from the geometric properties of the trapping horizon and its tangent vector, which can be related to the surface gravity of the event horizon. We are led to a generalization of the surface gravity to the dynamical cases without Killing vectors to

$$
\kappa_{A}^{2} \equiv-\frac{1}{2} \kappa \mathcal{E}_{-}
$$


although it is quite different from the generalizations of the surface gravity proposed in the literatures. In order to see if the definition above is appropriate, the relation to the surface gravity in more general geometries should be examined. This is left for future studies.

\section{Comments}

From the viewpoint of the observers on top of the trapping horizon in vacuum, we have shown that the energy-momentum tensor is always that of an ingoing negative energy flux at the speed of light. The negative vacuum energy accumulates behind the trapping horizon, so that the total energy decreases over time. As the black hole's mass decreases, the size of the "neck" $a(u)$ shrinks, while the interior space under the neck can still remain large. The geometry is reminiscent of "Wheeler's bag of gold". The collapsed matter (together with its information) stays in the bag.

The low-energy effective theory cannot reliably predict whether the size of the neck shrinks to 0 , and whether the bag detaches then. As long as the neck does not shrink to exactly 0 , the information inside the bag is still accessible to outside observers. However, from the viewpoint of distant observers, a black hole with a tiny neck is a remnant of tiny energy but huge entropy. Feynman diagrams involving these remnants are expected to contribute to scattering amplitudes due to the huge volume of phase space to be integrated over.

Furthermore, since macroscopic negative energy is allowed to accumulate, no one should expect the holographic principle to hold. For reviews on the information paradox, see e.g. refs. [8] and [9].

If the black-hole remnants are not acceptable, or if the holographic principle must hold, some of the assumptions we made in this paper must be invalid. In this paper, we assumed the presence of the time-like apparent horizon. We also assumed that gaugeinvariant combinations of the vacuum energy-momentum tensor is much smaller than that of classical matter such that the geometry is not modified by the quantum correction at the leading order of the perturbative expansion, namely, (3.2)-(3.4). However, the ingoing negative energy which is necessary for the time-like apparent horizon is as large as the energy of classical matter (but opposite in sign) from the viewpoint of an observer on the trapping horizon. It seems unavoidable to have some gauge-invariant quantities large in vacuum.

As we found that the quantum effect in the energy-momentum tensor becomes comparable to that of classical matter, the assumption that the quantum corrections must be very small in the gauge-invariant combinations (3.2)-(3.4) is no longer incontestable. For the sake of preserving the holographic principle, it might be preferable to impose the condition

$$
G_{\mu \nu} \xi^{\mu} \xi^{\nu}=\kappa T_{\xi \xi} \ll \mathcal{O}\left(\frac{1}{a^{2}}\right)
$$

instead of all of the conditions (3.2)-(3.4). Whether the holographic principle can hold relies on the properties of the vacuum energy-momentum tensor. For example, if it satisfies the null energy condition, there can be no trapping horizon because eq. (2.21) cannot be satisfied. The KMY model [10] (see also refs. [11]-[16]) is such a scenario. 
Recall that, for the Schwarzschild solution, the event horizon consists of the future horizon (i.e. the black-hole horizon) and the past horizon (i.e. the white-hole horizon). It is conventionally assumed that only the future horizon is relevant to the dynamical process of black-hole formation and evaporation. However, in the KMY model [10] and the model proposed in ref. [17], the white-hole horizon plays (at least conceptually) a crucial role. We apply the same calculation to these models in the appendix.

To summarize, different classes of vacuum energy-momentum tensor (corresponding to different classes of quantum field theories) lead to different models of black holes. Some of the models are compatible with the holographic principle, e.g. the fuzzballs $[18,19]$, the KMY model [10], the firewall [20-22], and the anti-podal identification [23], and other models are not compatible with the holographic principle (e.g. the conventional model). Yet regardless of whether you believe in the holographic principle, we emphasize the crucial role played by the quantum effect of the vacuum energy-momentum tensor.

\section{Acknowledgments}

The authors would like to thank Chong-Sun Chu, Hikaru Kawai, Samir Mathur, Yutaka Matsuo, Wen-Yu Wen, and Yuki Yokokura for discussions. The work is supported in part by the Ministry of Science and Technology, R.O.C. (project no. 107-2119-M-002-031MY3) and by National Taiwan University (project no. 105R8700-2). The work of Y.M. is supported in part by JSPS KAKENHI Grants No. JP17H06462.

\section{A An alternative scenario}

The dynamical analogue of the white-hole horizon is an anti-trapping horizon. At large distances where the curvature is small, we have eq. (2.5). We would have an anti-trapped region if

$$
\partial_{u} r>0, \text { and } \partial_{v} r>0
$$

in part of the spacetime. The boundary of the anti-trapped region is then an antitrapping horizon.

Here we consider alternative scenarios $[10,17]$ in which the anti-trapping horizon plays an important conceptual role in the following sense although there is actually no antitrapped region. There would be an anti-trapping horizon in the region occupied by the collapsing matter if the geometry is given by the analytic continuation of the geometry in vacuum outside the collapsing matter.

Let us first consider what would happen if there is actually an anti-trapping horizon. All we need to do is to carry out a time-reversal transformation, that is, to interchange $u$ and $v$ in our discussions above. Flipping the Penrose diagram figure 1, we find that for the anti-trapping horizon in figure 3.

The location of the anti-trapping horizon can be specified as $u=u_{0}(v)$ for a given function $u_{0}(v)$. Along the anti-trapping horizon, we must have

$$
\partial_{u} r=0,
$$




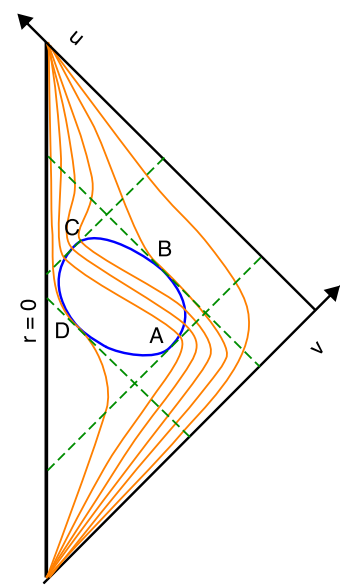

Figure 3. The schematic Penrose diagram with an anti-trapping horizon. The interpretation of this diagram is analogous to figure 1.

analogous to eq. (2.6). In particular, for the segment $B C$ in figure 3 , generically we have

$$
\partial_{u}^{2} r<0, \quad \partial_{v} \partial_{u} r<0,
$$

analogous to eq. (2.8).

The expansions (2.12), (2.13) are changed to

$$
\begin{aligned}
C(u, v) & =\bar{C}_{0}(v)+\bar{C}_{1}(v)\left(u-u_{0}(v)\right)+\frac{1}{2} \bar{C}_{2}(v)\left(u-u_{0}(v)\right)^{2}+\cdots, \\
r(u, v) & =\bar{a}(v)+\frac{1}{2} \bar{r}_{2}(v)\left(u-u_{0}(v)\right)^{2}+\cdots
\end{aligned}
$$

Following similar calculations we did above for the trapping horizon, we find that

$$
\bar{r}_{2}(v)<0 \quad u_{0}^{\prime}<0,
$$

implying that

$$
\bar{T}_{u u}^{(0)}(v)>0, \quad R_{\theta \theta}>1,
$$

where $\bar{T}_{u u}^{(0)}(v) \equiv T_{u u}\left(u_{0}(v), v\right)$, as necessary conditions for the existence of the segment $B C$ on the anti-trapping horizon. Due to eq. (A.6), this segment of the anti-trapping horizon is space-like, reflecting the fact that the inside of the anti-trapping horizon is an anti-trapped region.

As the condition (3.2) is necessarily violated, we can no longer use these conditions to estimate the energy-momentum tensor around the anti-trapping horizon. As the segment $B C$ of the anti-trapping horizon is space-like, in fact, shrinking faster than light, it is impossible for collapsing matter to fall into the trapped region from here.

Open Access. This article is distributed under the terms of the Creative Commons Attribution License (CC-BY 4.0), which permits any use, distribution and reproduction in any medium, provided the original author(s) and source are credited. 


\section{References}

[1] P.C.W. Davies, S.A. Fulling and W.G. Unruh, Energy momentum tensor near an evaporating black hole, Phys. Rev. D 13 (1976) 2720 [INSPIRE].

[2] R. Parentani and T. Piran, The internal geometry of an evaporating black hole, Phys. Rev. Lett. 73 (1994) 2805 [hep-th/9405007] [INSPIRE].

[3] P.-M. Ho and Y. Matsuo, On the near-horizon geometry of an evaporating black hole, JHEP 07 (2018) 047 [arXiv: 1804.04821] [INSPIRE].

[4] P.-M. Ho, Y. Matsuo and S.-J. Yang, Vacuum energy at apparent horizon in conventional model of black holes, arXiv:1904.01322 [INSPIRE].

[5] V.P. Frolov and G.A. Vilkovisky, Spherically symmetric collapse in quantum gravity, Phys. Lett. B 106 (1981) 307 [INSPIRE].

[6] T.A. Roman and P.G. Bergmann, Stellar collapse without singularities?, Phys. Rev. D 28 (1983) 1265 [INSPIRE].

[7] S.A. Hayward, Formation and evaporation of regular black holes, Phys. Rev. Lett. 96 (2006) 031103 [gr-qc/0506126] [INSPIRE].

[8] S.D. Mathur, The information paradox: a pedagogical introduction, Class. Quant. Grav. 26 (2009) 224001 [arXiv:0909.1038] [INSPIRE].

[9] D. Marolf, The black hole information problem: past, present and future, Rept. Prog. Phys. 80 (2017) 092001 [arXiv: 1703.02143] [INSPIRE].

[10] H. Kawai, Y. Matsuo and Y. Yokokura, A self-consistent model of the black hole evaporation, Int. J. Mod. Phys. A 28 (2013) 1350050 [arXiv:1302.4733] [InSPIRE].

[11] H. Kawai and Y. Yokokura, Phenomenological description of the interior of the Schwarzschild black hole, Int. J. Mod. Phys. A 30 (2015) 1550091 [arXiv:1409.5784] [INSPIRE].

[12] P.-M. Ho, Comment on self-consistent model of black hole formation and evaporation, JHEP 08 (2015) 096 [arXiv: 1505.02468] [INSPIRE].

[13] H. Kawai and Y. Yokokura, Interior of black holes and information recovery, Phys. Rev. D 93 (2016) 044011 [arXiv: 1509.08472] [INSPIRE].

[14] P.-M. Ho, The absence of horizon in black-hole formation, Nucl. Phys. B 909 (2016) 394 [arXiv: 1510.07157] [INSPIRE].

[15] P.-M. Ho, Asymptotic black holes, Class. Quant. Grav. 34 (2017) 085006 [arXiv: 1609.05775] [INSPIRE].

[16] H. Kawai and Y. Yokokura, A model of black hole evaporation and $4 D$ Weyl anomaly, Universe 3 (2017) 51 [arXiv:1701.03455] [INSPIRE].

[17] C.R. Stephens, G. 't Hooft and B.F. Whiting, Black hole evaporation without information loss, Class. Quant. Grav. 11 (1994) 621 [gr-qc/9310006] [INSPIRE].

[18] O. Lunin and S.D. Mathur, AdS/CFT duality and the black hole information paradox, Nucl. Phys. B 623 (2002) 342 [hep-th/0109154] [INSPIRE].

[19] O. Lunin and S.D. Mathur, Statistical interpretation of Bekenstein entropy for systems with a stretched horizon, Phys. Rev. Lett. 88 (2002) 211303 [hep-th/0202072] [INSPIRE].

[20] A. Almheiri, D. Marolf, J. Polchinski and J. Sully, Black holes: complementarity or firewalls?, JHEP 02 (2013) 062 [arXiv: 1207.3123] [INSPIRE]. 
[21] S.L. Braunstein, Black hole entropy as entropy of entanglement, or it's curtains for the equivalence principle, [arXiv:0907.1190v1].

[22] S.L. Braunstein, S. Pirandola and K. Życzkowski, Better late than never: information retrieval from black holes, Phys. Rev. Lett. 110 (2013) 101301 [arXiv:0907.1190] [INSPIRE].

[23] G. 't Hooft, What happens in a black hole when a particle meets its antipode, arXiv: 1804.05744 [INSPIRE]. 